\title{
Cannabis for the Treatment of Attention Deficit Hyperactivity Disorder: A Report of 3 Cases
}

\author{
Holly Mansella Declan Quinn ${ }^{b}$ Lauren E. Kelly ${ }^{c, d}$ Jane Alcorn ${ }^{a}$ \\ ${ }^{a}$ College of Pharmacy and Nutrition, University of Saskatchewan, Saskatoon, SK, Canada; ${ }^{b}$ Division of Child and \\ Adolescent Psychiatry, College of Medicine, University of Saskatchewan, Saskatoon, SK, Canada; 'Departments of \\ Pharmacology and Therapeutics and Community Health Sciences, University of Manitoba, Winnipeg, MB, Canada; \\ ${ }^{d}$ Children's Hospital Research Institute of Manitoba, Winnipeg, MB, Canada
}

\section{Keywords \\ Cannabis · Cannabinoid · Attention deficit hyperactivity disorder · Case report}

\begin{abstract}
Attention deficit hyperactivity disorder (ADHD) is a chronic neurobehavioral disorder that is highly prevalent in children and adults. An increasing number of patients with ADHD are self-medicating with cannabis, despite a lack of evidence on efficacy and safety. This case report describes 3 males (ages 18,22 , and 23) who have integrated cannabis into their treatment regimen with positive results. Semistructured interviews conducted with the patients describe subjective improvements in symptoms and on quality of life. Improvements on validated rating scales conducted post-cannabis initiation, compared to pre-cannabis initiation obtained from the medical chart, corroborated their personal accounts. Scores on the PHQ-9 (measuring depression) improved by $8-22$ points (30-81\%), and the SCARED (measuring anxiety) ranged from 0 to 27 points (up to 33\%). Improvements on the CEER- 9 scale (measuring regulation) ranged from 2 to 7 points (22-78\%), and the 9 -item SNAP scale (measuring inattention) showed improvements of 2-8 points (7-
\end{abstract}

(C) 2022 The Author(s)

Published by S. Karger AG, Basel

This is an Open Access article licensed under the Creative Commons Attribution-NonCommercial-4.0 International License (CC BY-NC) (http://www.karger.com/Services/OpenAccessLicense), applicable to the online version of the article only. Usage and distribution for commercial purposes requires written permission.
30\%). Mild adverse events including short-term memory problems, dry mouth, and sleepiness were reported. Blood samples were also collected from the patients to determine the plasma concentrations of the cannabinoids and relevant metabolites before and after a cannabis administration. After cannabis use, the plasma levels for CBD and THC ranged from 0 to $15.29 \mathrm{ng} / \mathrm{mL}$ and 1.32 to $13.76 \mathrm{ng} / \mathrm{mL}$, respectively. Cannabinoids, however, were not detected prior to dosing, suggesting that cannabis played a complimentary role in the therapeutic regimen of these 3 patients. Clinical trials are recommended to confirm the efficacy of cannabis in the treatment of ADHD.

(c) 2022 The Author(s).

Published by S. Karger AG, Basel

\section{Introduction}

Public acceptance of medical cannabis for the treatment of chronic conditions has increased despite a lack of scientific evidence for safety and efficacy $[1,2]$. In Cana$\mathrm{da}$, access to both medical and recreational cannabis is now legal, and self-medication with cannabis for a variety of symptoms has become more prevalent. Evidencebased information has not kept pace with the legislation, 
and many clinicians remain reluctant to authorize cannabis until robust evidence is available to guide treatment [3]. Unfortunately, the limited evidence base may persist due to key challenges such as the difficulty in conducting randomized and placebo-controlled trials with cannabis [4] and the significant variability in cultivars. With $>489$ distinct compounds in the leaves and flowering tops of cannabis plants, which include at least 120 different phytocannabinoids and other entities such as flavonoids and terpenes $[5,6]$, cultivars demonstrate important differences in therapeutic effect. Even within a given cultivar, soil and climate conditions and various cultivation techniques can influence its constituent bioactive components. The method of consumption or route of administration further impacts the pharmacokinetics and therapeutic effects of cannabis [7].

Attention deficit hyperactivity disorder (ADHD) is one such condition where few clinicians authorize cannabis, but interest in self-medication is high. ADHD is a chronic neurobehavioral disorder characterized by inattention and/or hyperactivity-impulsivity with a prevalence of approximately $7 \%$ in children and $2.5 \%$ in adults $[8,9]$. Over half of the children and adults with ADHD have comorbid psychiatric conditions, such as sleep disorders, mood and anxiety disorders, and oppositional defiant disorder $[10,11]$. In addition to the first-line pharmacotherapy with stimulants, internet discussions frequently indicate and advocate use of cannabis for relief of ADHD symptoms [12-14]. In one study, at least 3 times as many online comments attested for its therapeutic benefits in comparison with harm or lack of efficacy [14]. Despite the public interest and anecdotal reports, we only found one study on the use of cannabis in ADHD which compared Sativex Oromucosal Spray to placebo in a pilot study of 30 adults, where participants in the intervention group showed no difference in the primary outcome (cognitive performance as measured by the QbTest), but improved symptoms of hyperactivity/impulsivity (secondary outcome, $p=0.03$ ) [15]. While this study investigated a commercially available product (containing 1:1 cannabidiol $[\mathrm{CBD}]: \Delta^{9}$-tetrahydrocannabinol $[\mathrm{THC}]$ ), a product available in some countries by prescription, many patients are self-medicating with unlicensed products in a variety of formulations [12-14].

In the absence of scientific data, case reports are valuable for presenting new observations, generating hypotheses, and providing in-depth examinations of a subject of study as well as its related contextual conditions [16]. In this case report, we identified patients from a psychiatry practice who were prescribed ADHD medications but also self-medicated with cannabis. We explored treatment efficacy and patient perceptions of cannabis use. Since no literature exists to provide guidance for cannabis dosing in patients with ADHD, we also collected patient blood samples to determine plasma concentrations of the cannabinoids and relevant metabolites.

\section{Methods}

Three patients who were taking cannabis for symptomatic relief of their ADHD participated in telephone interviews conducted by one of the authors (H.M.). The patients were previously diagnosed using the DSM-V criteria. The interviews lasted between 39 and $59 \mathrm{~min}$. To characterize the patient's experiences of taking cannabis and its perceived efficacy, a semistructured interview guide facilitated a discussion about the patient's life prior to initiating cannabis, the decision/reasons for starting it, how (if at all) things have changed since initiating cannabis, logistics on consumption and access, and what (if any) concerns they might have about cannabis. A cannabis side effect survey [17] inquired about potential cannabis side effects experienced by the patients, using the time frame of the previous week.

Self-report symptom rating scales, including the Swanson Nolan and Pelham (SNAP-IV) (90-item) [18], the Screen for Child Anxiety Related Emotional Disorders (SCARED) [19], and Patient Health Questionnaire (PHQ-9) [20], were performed during the interviews as a measure for treatment efficacy. The SNAP-IV rating scale contains 90 items from the DSM-IV criteria which assess inattention, hyperactivity/impulsivity, and oppositional defiant disorder, as well as items representing a general index of childhood problems. This tool, which is a modified version of the original SNAP questionnaire $[18,21]$, consists of Likert scale questions that measure the frequency/severity of a symptom or behavior. The items are scaled from 0 to 3 ( 0 = "not at all," and 3 = "very much"), and the tool has had good reliability and validity in different study samples [22]. The SCARED rating scale and PHQ-9 assessed symptoms of anxiety and depression, respectively. The 3 rating scales were summed according to standardized methods [18-20] to achieve a composite score, with higher scores equating to higher levels of debility. Baseline symptom scores obtained before cannabis was initiated were collected from the patient's medical chart and subtracted from the totals, producing an indicator of symptom change. ADHD inattentive symptoms were measured using items 1-9 of the SNAP-IV, while emotional regulation and irritability were measured using the Clinical Evaluation of Emotional Regulation-9 (CEER-9) method, whereby items 21, 23, 25, 26, 28, 34, 38, 39 , and 54 from the SNAP-IV questionnaire were converted to a binary score ( 1 = not at all; $2=$ very much) [23].

Two blood samples were obtained from each patient by the mobile laboratory to allow quantification of plasma concentrations of cannabinoids and relevant metabolites (THC, CBD, 11-hydroxy-THC, 7-hydroxy-CBD, cannabichromene, and 11-nor-9-carboxy-tertrahydrocannabinol). The samples were collected in BD Vacutainer ${ }^{\circledR}$ Barricor ${ }^{\mathrm{TM}}$ tubes [24], centrifuged to separate plasma, transferred to Eppendorf ${ }^{\mathrm{TM}}$ Protein LoBind microcentrifuge tubes, and stored in $\mathrm{a}-80^{\circ} \mathrm{C}$ freezer until they were analyzed by liquid chromatography-tandem mass spectrometry. 
Table 1. Rating scale scores obtained from 3 ADHD patients' medical charts prior to and after initiation of cannabis

\begin{tabular}{|c|c|c|c|c|c|c|c|}
\hline \multirow[t]{2}{*}{ Patient } & \multicolumn{2}{|c|}{ Construct measured } & \multirow{2}{*}{$\begin{array}{l}\text { PHQ-9 } \\
\text { depression } \\
{[0-27]}\end{array}$} & \multirow{2}{*}{$\begin{array}{l}\text { SCARED } \\
\text { anxiety } \\
{[0-82]}\end{array}$} & \multirow{2}{*}{$\begin{array}{l}\text { CEER-9 } \\
\text { emotional regulation } \\
{[0-9]}\end{array}$} & \multirow{2}{*}{$\begin{array}{l}\text { SNAP } \\
\text { inattention } \\
{[0-27]}\end{array}$} & \multirow{2}{*}{$\begin{array}{l}\text { SNAP-IV (90-item) } \\
\text { multiple } \\
{[0-180]}\end{array}$} \\
\hline & time frame & date & & & & & \\
\hline \multirow[t]{2}{*}{1} & Pre-cannabis & Mar 2020 & 22 & 77 & 9 & 24 & $\mathrm{n} / \mathrm{a}$ \\
\hline & Post-cannabis & Jul 2021 & 0 & 50 & 2 & 16 & 127 \\
\hline & Post-cannabis & May 2021 & 1 & 19 & 4 & 9 & 57 \\
\hline \multirow[t]{3}{*}{3} & Pre-cannabis & Mar 2020 & 13 & 30 & 5 & 7 & $\mathrm{n} / \mathrm{a}$ \\
\hline & & Jun 2020 & $\mathrm{n} / \mathrm{a}$ & $\mathrm{n} / \mathrm{a}$ & 3 & 18 & $\mathrm{n} / \mathrm{a}$ \\
\hline & Post-cannabis & Jul 2021 & 5 & 30 & 0 & 5 & 46 \\
\hline
\end{tabular}

PHQ-9, Patient Health Questionnaire-9; SCARED, Screen for Child Anxiety Related Emotional Disorders; CEER-9, Clinical Evaluation of Emotional Regulation-9; SNAP-IV (90-item), Swanson Nolan and Pelham rating scale; n/a, data not available in chart.

The method was previously developed and validated within our institution [25] according to FDA guidelines [26]. Written informed consent was obtained from patients for publication of the details of their medical case, and ethical approval for data collection was approved by the Biomedical Research Ethics Boards at the University of Saskatchewan (\#1726).

\section{Case Presentations and Patient Perceptions of Cannabis}

The case presentations as described in this section represent an account of the patients' testimonials, with information verified from the medical charts. The patients had the opportunity to review these descriptions for accuracy prior to publication.

\section{Patient 1}

A 23-year-old white male with ADHD and generalized anxiety disorder decided to use cannabis after learning of its effectiveness for $\mathrm{ADHD}$ online. Medicated with methylphenidate, pregabalin, fluoxetine, and clonidine, he first tried cannabis in his teens. $\mathrm{He}$ used it periodically as cannabis improved his focus. He eventually consulted his physician and received an authorization for medical cannabis in a CBD:THC 20:1 ratio, twice daily. He currently alternates between taking cannabidiol oil orally or smoking flower or bud. Although he realizes oil is less harmful for his lungs and more suitable in some situations, he feels smoking is more relaxing. He describes cannabis as a "really good helping hand" to compliment his other medications, and finds he is more open with others, less anxious, and his emotions are less exaggerated. Cannabis has improved his ability to maintain focus. Regarding his life before cannabis, he says "I was all over the place, bouncing off the walls kind of thing. Couldn't really stay on one task for long. I'd get halfway to three quarters done one task and then move on and do a completely different task." Cannabis, as he describes, "levels him out," and he can complete tasks more efficiently. Although his family members' reaction to his cannabis have been mixed (with some individuals very supportive and others skeptical), he believes they have all noted a positive impact on his demeanor. Since taking cannabis regularly, he has found and maintained successful employ- ment at a job away from home. The cost of medical cannabis is unaffordable, so he obtains recreational cannabis at half the cost.

\section{Patient 2}

A Caucasian male with ADHD has taken methylphenidate off and on since the third grade. He disliked being medicated with stimulants because he felt they changed his personality, and he continued to struggle with emotional regulation. At age 17, he was prescribed lithium, and he decided to start cannabidiol oil (CBD:THC in a 20:1 ratio) once daily (at bedtime) after a family member recommended it to him. Taking cannabis, he says, makes him feel more relaxed and helps him to focus and to feel more "himself." The combination of lithium (300 mg at bedtime), cannabis ( $1 \mathrm{~mL}$ at bedtime), and a good support system he says have completely changed his life. Previously he lacked motivation, did not do well in school, and was admitted for psychiatric care. Since starting cannabis, he was successfully weaned off his other medications for ADHD (takes only lithium for depression and cannabis) and has completely turned his life around. Regarding his life before cannabis he says, "I was definitely a lot more jittery and stuff when I was in school...It [cannabis] helped me to focus a lot more, and it helped me ease off my ADHD meds actually." He works full time and runs a business, sets long-term goals for future, and has made many new friends, which has improved his social life.

\section{Patient 3}

A 22-year-old male was diagnosed with ADHD when he was 20 and started self-medicating with cannabis. He had no prior history of cannabis use, but with dispensaries opening in Canada and a family member who was considering it for anxiety, he decided to try it as well. He feels that cannabis calms him, helps him to slow down and focus on one thing, and to sleep at night. He believes that cannabis works synergistically with his other medications (dextroamphetamine, amantadine, and pregabalin) to improve his concentration and control his racing thoughts, anxiety, and emotions. Prior to treatment with this combination and as a child, he describes, "the lashing out was really bad, not listening to adult fig- 
Table 2. Plasma concentrations of cannabinoids and relevant metabolites obtained from blood samples of 3 ADHD patients self-medicating with cannabis

\begin{tabular}{|c|c|c|c|c|c|c|c|c|c|}
\hline Patient & $\begin{array}{l}\text { Self-reported product } \\
\text { and route }\end{array}$ & Patient sample & $\begin{array}{l}\text { CBD, ng/ } \\
\mathrm{mL}\end{array}$ & $\begin{array}{l}\text { 6-OH-CBD, } \\
\mathrm{ng} / \mathrm{mL}\end{array}$ & $\begin{array}{l}\text { 7-OH-CBD, } \\
\mathrm{ng} / \mathrm{mL}\end{array}$ & $\begin{array}{l}\text { THC, ng/ } \\
\mathrm{mL}\end{array}$ & $\begin{array}{l}\text { 11-OH-THC, } \\
\mathrm{ng} / \mathrm{mL}\end{array}$ & $\begin{array}{l}\mathrm{THC}-\mathrm{COOH}, \\
\mathrm{ng} / \mathrm{mL}\end{array}$ & $\begin{array}{l}\mathrm{CBC} \\
\mathrm{ng} / \mathrm{mL}\end{array}$ \\
\hline \multirow[t]{2}{*}{1} & \multirow{2}{*}{$\begin{array}{l}\text { CBD:THC 20:1 flower } \\
\text { smoked }\end{array}$} & Pre-dose & ND & ND & ND & ND & ND & ND & ND \\
\hline & & Post-dose (2 h) & BLOQ & ND & ND & 13.76 & 6.30 & 248.56 & ND \\
\hline 2 & $\begin{array}{l}\text { CBD:THC } \\
\text { 20:1 oil } 1 \mathrm{~mL} \\
\text { Oral }\end{array}$ & Pre-dose & ND & ND & ND & ND & ND & ND & ND \\
\hline \multirow[t]{2}{*}{3} & \multirow{2}{*}{$\begin{array}{l}\text { CBD:THC } \\
\text { 0:19 flower smoked }\end{array}$} & Pre-dose & ND & ND & ND & ND & ND & ND & ND \\
\hline & & Post-dose (30 min) & ND & ND & ND & 7.65 & 0.60 & 14.51 & ND \\
\hline
\end{tabular}

BLOQ, below the limit of quantification; ND, not detected.

ures. And emotions...And forgetting where I placed stuff. I lost my wallet and keys a lot growing up." Having experimented with various strains, ratios, and methods of consumption, he prefers an Indica blend that is higher in THC and lower in CBD. Sativa caused him to be a bit more hyperactive and increased his anxiety, whereas an Indica dominant blend helps him to cool down. He is currently smoking a product that has a CBD:THC ratio of 0:18-19 at bedtime, and says that this formulation does not make him feel intoxicated. Dabbing (which has a higher concentration of THC), however, has too much THC and puts him in an "unmotivated funk." He has tried edibles and oils, but they fail to improve his sleep. He avoids vaping because with his addictive personality and convenience of vaping, he found that he vaped more than just at night. He obtains his supply from a recreational cannabis dispensary where it is most affordable.

Rating Scale Changes before and after Initiation of Cannabis

Table 1 presents the rating scales obtained from the patient's medical record and on the day of the interview. These scores were (respectively) obtained before and after the patients used cannabis regularly. Consistent with the testimonials, all 3 patients experienced positive improvements on the measures for depression, emotional regulation, and inattention. The scores from patients 1 and 2 also indicate an improvement in the SCARED, which measures symptoms of anxiety.

\section{Cannabis Side Effects}

All patients reported mild side effects from cannabis use. Patient 2, who consistently takes oil orally, experienced short-term memory problems. Patients 1 and 3, who alternate between oral and inhalation routes, reported dry mouth and sleepiness. Patient 1 reported occasional experiences of constant desire and more forgetfulness and apathy, and patient 3 reported an altered sense of time.

\section{Blood Sample Analysis}

Two blood samples were taken at home just prior to and $2 \mathrm{~h}$ after cannabis administration from each patient to quantify plasma concentrations of major cannabinoids and relevant metabolites
(Table 2). Each patient self-reported their cannabis product and was not verified by chemical analysis. None of the patients had detectable plasma concentrations of any of the cannabinoids tested during the pre-dose level (trough), which represents the time when plasma levels are the lowest. The post-dose level, which is intended to represent the maximum plasma cannabinoid concentration, should be collected at approximately $30 \mathrm{~min}$ after smoked cannabis and $2 \mathrm{~h}$ for edible formulations [6]. Patient 1 , who alternates between routes, ended up smoking cannabis on the day the mobile lab was scheduled to draw levels at $2 \mathrm{~h}$ later. Therefore, patient 1 's plasma concentrations are not representative of the true maximum.

\section{Discussion/Conclusion}

We describe 3 patients with ADHD who added cannabis to their treatment regimen and experienced positive therapeutic effects. The improvements in their symptoms and quality of life were substantial, such as the ability to keep emotions in check ( 3 patients) or to obtain and excel at a new job with more responsibility (2 patients). Objective measures accompanied these narratives with all 3 patients experiencing improvements in validated rating scales for measures of mental health. Scores on the PHQ9 , which measure depression, improved by $8-22$ points (30-81\%). Improvements on the SCARED, which measure anxiety, ranged from 0 to 27 (up to $33 \%$ ), and the CEER-9 scale, which indicates emotional regulation, ranged from 2 to 7 (22-78\%). Finally, the 9-item SNAP scale measuring inattention showed improvements ranging from 2 to 8 points on the raw scale, which equated to $7-30 \%$.

Notably, all 3 patients used cannabis as an adjunct to their other medications (e.g., stimulants, antidepressants, 
or mood stabilizers). Patient 1 described cannabis as "a really good helping hand" to compliment his other medications. Patient 3 was able to discontinue his stimulant pharmacotherapy, but acknowledged that the "cannabis, in addition to a change in prescription medications to lithium, helped to change his life." Plasma levels of the cannabinoids were not detectable at trough levels suggesting that the effects of the cannabis may not be sustained throughout the day; however, the patients perceived the effects of the cannabis to last throughout the day. This bespeaks to the importance of the other medications to ensure treatment success.

All 3 patients discussed the use of cannabis with their psychiatrist and were authorized oral cannabidiol oil CBD:THC (20:1) from a medical source. Our patient interviews and accompanying blood levels, however, indicated that this route and formulation was not consistent, and that the patients sought out a product and regimen that worked for them. Two patients reported taking cannabis once daily, whereas the other patient preferred a twice daily regimen. Two patients preferred smoking cannabis (as opposed to an edible oil). With respect to chemical composition, 1 patient preferred a product high in THC and low in CBD (CBD:THC 0:18-19). Another patient reported using CBD:THC 20:1, but high amounts of THC and no CBD were detected in the post-dose sample, which brings into question the contents of product used by the patient. The use of cannabis is also complicated by the dual supply streams in Canada. Two patients obtained their cannabis from a recreational cannabis store, versus a medical source due to significant cost differences (double the price for medical cannabis). Unlike other medications, patients are freely available to choose their cannabis product and source. Even within the same source, batch to batch variation is expected, which contributes to significant inter- and intrapatient variability with cannabis-based medicine.

An increasing number of patients are self-medicating with cannabis for the treatment of ADHD. Yet, a dearth of literature exists on this topic, and only 2 other published case reports of patients receiving benefits from cannabis were found in the literature. One publication from 2008 reported improvement in performance tests (ART2020 and TAP) in an adult male smoking cannabis, suggesting that THC may have atypical effects in patients with ADHD [27]. A second case report in 2018 described improved symptoms in an ADHD patient who was taking a product high in THC [28]. Although the potential mechanism of cannabis remains to be determined, cannabinoids, such as THC, may mediate their effects through induction of dopamine release in the human striatum $[28,29]$. Elucidating such mechanisms will be particularly challenging since the biochemical composition of cannabis (e.g., phytocannabinoids, terpenes, and flavonoids) varies from strain to strain. Plasma cannabinoid concentrations of 2 of the patients in the present discussion were high in THC (with no CBD), while the other patient had higher CBD concentrations, but THC was still present. In the previous literature, products high in THC or containing both THC and CBD ameliorated ADHD symptoms $[15,27,28]$. While we cannot draw any conclusions on the optimal ratio of CBD:THC, we suggest that future studies involve a product consisting of some amount of THC (instead of a product consisting only of CBD).

An important limitation of case reports is their inability to generalize to a wider population given the selection bias, and there is limited information that can be gleaned from these reports of a few selected patients. While randomized controlled trials are urgently needed to provide insight on the efficacy of cannabis in the treatment of ADHD, such studies will never be performed with smoked cannabis flower, and we are left to rely on observational data for these exposures. This report adds to the literature by providing detailed personal accounts from 3 patients and objective evidence of improvement on validated measures for ADHD symptoms. Clinicians who care for patients who self-medicate with cannabis should aim to objectively monitor symptoms, using validated scales for $\mathrm{ADHD}$ and other comorbidities. The patients presented in this case report were also taking concurrent medications; therefore, the added benefits of cannabis on ADHD symptoms are unclear. Significant variation noted amongst these 3 individuals (e.g., product, chemical composition, dose, route of administration, and supplier) highlights the challenges with cannabis-based medicine development and research.

\section{Acknowledgments}

The authors would like to thank Lorraine Sadler with TLC Mobile Lab and Stephanie Vuong for collection and analysis of blood samples, respectively.

\section{Statement of Ethics}

Written informed consent was obtained from patients for publication of the details of their medical case, and ethical approval for data collection was approved by the Biomedical Research Ethics Boards at the University of Saskatchewan (\#1726). 


\section{Conflict of Interest Statement}

The authors have no conflicts of interest to declare.

\section{Funding Sources}

This work was funded by an internal College of Pharmacy and Nutrition Seed Fund Grant.

\section{Author Contributions}

H.M. contributed to the research design, data collection, and manuscript writing. D.Q., L.E.K., and J.A. contributed to the research design and reviewed and revised the manuscript.

\section{Data Availability Statement}

The data that support the findings of this case report are not publicly available to protect the individual's anonymity.

\section{References}

1 Sznitman SR, Bretteville-Jensen AL. Public opinion and medical cannabis policies: examining the role of underlying beliefs and national medical cannabis policies. Harm Reduct J. 2015 Oct 14;12:46.

2 Dai H, Richter KP. A national survey of marijuana use among us adults with medical conditions, 2016-2017. JAMA Netw Open. 2019;2(9) e1911936.

3 Ng JY, Gilotra K, Usman S, Chang Y, Busse JW. Attitudes toward medical cannabis among family physicians practising in Ontario, Canada: a qualitative research study. CMAJ Open. 2021 Apr 13;9(2):E342-8.

4 National Academies of Sciences, Engineering, and Medicine; Health and Medicine Division Board on Population Health and Public Health Practice; Committee on the Health Effects of Marijuana: An Evidence Review and Research Agenda. 15, challenges and barriers in conducting cannabis research. The health effects of cannabis and cannabinoids: the current state of evidence and recommendations for research. Washington, DC: National Academies Press (US); 2017 Jan 12. Available from: https://www.ncbi.nlm.nih.gov/books/ NBK425757/.

5 Morales P, Hurst DP, Reggio PH. Molecular targets of the phytocannabinoids: a complex picture. Prog Chem Org Nat Prod. 2017;103:10331

6 Health Canada. Information for health care professionals: cannabis (marihuana, marijuana) and the cannabinoids. Health Canada; 2013 [cited 2019 Nov 29]. Available from: www.canada. $\mathrm{ca} /$ content/dam/hc-sc/migration/hc-sc/dhpmps/alt formats/pdf/marihuana/med/infoprof-eng.pdf.

7 Grotenhermen F. Pharmacokinetics and pharmacodynamics of cannabinoids. Clin Pharmacokinet. 2004;42(4):327-60.

8 Thomas R, Sanders S, Doust J, Beller E, Glasziou P. Prevalence of attention-deficit/hyperactivity disorder: a systematic review and meta-analysis. Pediatrics. 2015;135:e994-1001.

9 Simon V, Czobor P, Bálint S, Mészáros A, Bitter I. Prevalence and correlates of adult attentiondeficit hyperactivity disorder: meta-analysis. $\mathrm{Br}$ J Psychiatry. 2009;194(3):204-11.

10 Katzman MA, Bilkey TS, Chokka PR, Fallu A, Klassen LJ. Adult ADHD and comorbid disor- ders: clinical implications of a dimensional approach. BMC Psychiatry. 2017 Aug 22;17(1): 302.

11 Reale L, Bartoli B, Cartabia M, Zanetti M, Costantino MA, Canevini MP, et al. Comorbidity prevalence and treatment outcome in children and adolescents with ADHD. Eur Child Adolesc Psychiatry. 2017 Dec;26(12): 1443-57.

12 Emanuel N. We give CBD oil to our sons with ADHD. 2021 [cited 2019 Nov 6]. Available from: https://www.scarymommy.com/adhd-cbd-oil/.

13 Children and adults with attention-deficit/hyperactivity disorder (CHADD). CBD oil and ADHD management: too big a leap. ADHD Weekly. 2018 [cited 2019 Nov 6]. Available from. https://chadd.org/adhd-weekly/cbd-oiland-adhd-management-too-big-a-leap/.

14 Mitchell JT, Sweitzer MM, Tunno AM, Kollins $\mathrm{SH}, \mathrm{McClernon}$ FJ. "I use weed for my ADHD": a qualitative analysis of online forum discussions on cannabis use and ADHD. PLoS One. 2016; 11(5):e0156614.

15 Cooper RE, Williams E, Seegobin S, Tye C, Kuntsi J, Asherson P. Cannabinoids inattention-deficit/hyperactivity disorder: a randomised-controlled trial. Eur Neuropsychopharmacol. 2017; 27:795-808.

16 Nissen T, Wynn R. The clinical case report: a review of its merits and limitations. BMC Res Notes. 2014 Apr 23;7:264.

17 Sexton M, Cuttler C, Mischley LK. A survey of cannabis acute effects and withdrawal symptoms: differential responses across user types and age. J Altern Complement Med. 2019;25: 326-35.

18 Swanson J. Clinician-administered screening tools [Internet]. Collaborative mental health care. The SNAP-IV teacher and parent rating scale [cited 2020 Jan 8]. Available from: http:// www.shared-care.ca/files/SNAP_IV_Long_ with_Scoring.pdf

19 Birmaher B, Brent DA, Chiappetta L, Bridge J, Monga S, Baugher M. Psychometric properties of the Screen for Child Anxiety Related Emotional Disorders (SCARED): a replication study. J Am Acad Child Adolesc Psychiatry. 1999; 38(10):1230-6.

20 Kroenke K, Spitzer RL, Williams JB. The PHQ-9: validity of a brief depression severity measure. J Gen Intern Med. 2001;16:606-13.
21 Swanson JM, Sandman CA, Deutsch C, Baren M. Methylphenidate hydrochloride given with or before breakfast: I. Behavioral, cognitive, and electrophysiological effects. Pediatrics. 1983;72: 49-55.

22 Bussing R, Fernandez M, Harwood M, Hou W, Garvan CW, Eyberg SM, et al. Parent and teacher Snap-IV ratings of attention deficit hyperactivity disorder symptoms: psychometric properties and normative ratings from a school district sample. Assessment. 2008;15(3):317-28.

23 Pylypow J, Quinn D, Duncan D, Balbuena L. A measure of emotional regulation and irritability in children and adolescents: the Clinical Evaluation of Emotional Regulation-9. J Atten Disord. 2020 Dec;24(14):2002-11.

24 Vuong S, Michel D, Alcorn J, Jane H, Tang-Wai

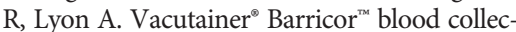
tion tube is the tube of choice for LC-MS/MS analysis of bioactive cannabinoids in plasma 2017. White paper. Available from: https://research-groups.usask.ca/cris/documents/Bioactive \% 20 Cannabinoids \% 20 Barricor $\% 20$ Tube\%20Study\%20Report.pdf.

25 Vuong S, Michel D, Huntsman R, Tang-Wai R, Wu F, Lyon AW, et al. Development of a liquid chromatography-tandem mass spectrometry assay for analysis of bioactive cannabinoids in plasma of pediatric patients on cannabis oil therapy. MSACL 2018 US 10th Annual Conference; 2018 Jan 21-25. Palm Springs, CA: 2018. p. 137.confpap

26 Food and Drug Administration (FDA); Center for Drug Evaluation Research (CDER); Center for Biologics Evaluation and Research (CBER). General considerations for pediatric pharmacokinetic studies for drugs and biological products. Draft guidance for industry. 1998.

27 Strohbeck-Kuehner P, Skopp G, Mattern R Cannabis improves symptoms of ADHD. Cannabinoids. 2008;3(1):1-3.

28 Hupli AMM. Medical cannabis for adult attention deficit hyperactivity disorder: sociological patient case report of cannabinoid therapeutics in Finland. Med Cannabis Cannabinoids. 2019; 1(2):112-8.

29 Bossong $\mathrm{MG}$, van Berckel BN, Boellaard $\mathrm{R}$, Zuurman L, Schuit RC, Windhorst AD, et al. Delta 9-tetrahydrocannabinol induces dopamine release in the human striatum. Neuropsychopharmacology. 2009 Feb;34(3):759-66. 